\title{
Análise Experimental de Sensoriamento de Espectro baseado em Detecção de Energia com a utilização de Wavelets
}

\author{
Erika Portela Lopes de Almeida ${ }^{1}$, Paulo Henrique Portela de Carvalho ${ }^{1}$, Pedro Antero Braga \\ Cordeiro $^{1}$ e Robson Domingos Vieira ${ }^{2}$ \\ ${ }^{1}$ Universidade de Brasília, Departamento de Engenharia Elétrica, Brasília-DF \\ ${ }^{2}$ Instituto Nokia de Tecnologia, Brasília-DF
}

\begin{abstract}
Resumo - O sensoriamento de espectro é uma das etapas principais de funcionamento das redes de rádio cognitivo. Neste artigo, o sensoriamento de espectro é investigado através de uma técnica de deteç̧ão de energia com a utilização de wavelets. Para analisar a confiabilidade da técnica, foram realizadas medidas na faixa de radiodifusão FM $(98 \mathrm{MHz})$ e na faixa de $800 \mathrm{MHz}$. Por fim, modificações na técnica original de deteç̧ão foram propostas a fim de melhorar o desempenho do algoritmo em situações reais.
\end{abstract}

Palavras-Chave - Rádio cognitivo, sensoriamento de espectro, detecção de energia e transformada wavelet .

Abstract - Spectrum sensing is a key functionality in cognitive radio networks. In this paper, spectrum sensing is studied with an energy detection technique, based on the Wavelet Transform. To evaluate the performance and the reliability of the original method, measurements on the FM broadcast $(98 \mathrm{MHz})$ band were made. Some changes in the original technique were proposed to improve the spectrum sensing reliability in real situations.

\section{INTRODUÇÃO}

O interesse pela tecnologia de rádio cognitivo tem crescido continuamente nos últimos anos. No início, a pesquisa em torno do conceito de rádio cognitivo [1] foi desenvolvida pelas universidades e institutos de pesquisas. Porém, atualmente grandes consórcios, financiados pela União Européia e que possuem a participação da indústria, estão sendo desenvolvidos com o objetivo de definir o conceito de uma rede de rádio cognitivo baseada no aumento da percepção e autonomia dos terminais. O próprio IEEE possui um comitê, Standards Coordinating Comittee 41 Dynamic Spectrum Access Networks, com o objetivo de avaliar projetos nas áreas de acesso dinâmico ao espectro, rádio cognitivo e outras tecnologias para redes da próxima geração. O principal motivo do aumento desse interesse é a diminuição do espectro disponível, que é visto como crítico para o desenvolvimento de novas tecnologias e negócios. Conseqüentemente, surge a necessidade de buscar novas soluções que possam explorar o limitado espectro existente de uma forma eficiente.

Desse modo, as redes de rádio cognitivo surgem com o intuito de utilizar o espectro de forma eficiente, otimizando o uso do espectro limitado disponível. Nas redes de rádio cognitivo, os dispositivos serão autônomos, capazes de otimizar o uso do recurso de acordo com as suas necessidades individuais e acessar o espectro licenciado de forma oportunista sem interferir com os usuários já existentes [1, 2, 3].

Por outro lado, a viabilidade das redes de rádio cognitivo tem sido questionada, especialmente no que diz respeito à inteligência e à percepção dos terminais, já que os mesmos devem ser móveis e dependentes de baterias. Além disso, requisitos de qualidade de serviço, sensoriamento de uma largura grande de espectro e atualização dinâmica das informações são desafios que devem ainda ser superados.

Em [3] são apresentados os princípios de funcionamento de uma rede de rádio cognitivo. Em resumo, inicialmente, o sensoriamento do espectro é realizado a fim de determinar a porção do espectro disponível para a transmissão. A identificação das porções disponíveis do espectro já inclui a verificação da existência de usuários licenciados. O próximo passo é escolher o melhor canal disponível. As etapas de gerenciamento e divisão do espectro cruzam as informações dos canais disponíveis com as necessidades dos usuários, só então é permitido o acesso de usuários não-licenciados. Caso a presença do usuário licenciado seja detectada ao longo do gerenciamento da conexão, o usuário não-licenciado desocupa o canal.

$\mathrm{Na}$ etapa de sensoriamento do espectro, o ambiente de propagação, mais especificamente a largura de espectro a ser inspecionado, é verificado. Assim, canais livres em um determinado instante são determinados. Para que uma técnica de sensoriamento seja eficiente, é necessário que ela perceba as alterações no ambiente de transmissão de forma rápida e confiável.

As técnicas de sensoriamento podem ser dividas em três classes: detecção no transmissor, detecção cooperativa e a detecção baseada em interferência [3]. Dentre as técnicas de detecção no transmissor, destacam-se a detecção de energia [4,5,6], detecção por filtro casado, detecção baseada nas propriedades de estacionariedade cíclica [7] e detecção não paramétrica de correlação cíclica .

Podem-se destacar algumas formas de detecção de energia. A primeira consiste em um banco de filtros seletivos na entrada do receptor [9]. Cada filtro seleciona um canal e a partir dessa informação calcula-se a energia. Como se pode perceber, essa é uma alternativa pouco flexível. Outra técnica conhecida de detecção de energia utiliza a informação de um períodograma para calcular, através do 
quadrado da magnitude da FFT, a energia dentro da banda escolhida para realizar o sensoriamento. Como abordado em [10], o períodograma é um estimador enviesado e inconsistente para o espectro de potência.

Nos últimos anos, contudo, tem-se iniciado o estudo da transformada wavelet [11] como uma alternativa aos dois métodos apresentados, tanto devido a sua flexibilidade, quanto à sua rapidez [4].

Entretanto, nenhum estudo que avalie o desempenho dessa última técnica em relação a sinais reais é encontrado, atualmente, na literatura. O objetivo desse trabalho é, portanto, avaliar experimentalmente a performance de uma técnica de detecção de energia com utilização de wavelets e propor alterações que permitam que a técnica seja viável em situações reais. Utilizou-se como base para o estudo, o método de detecção apresentado em [4].

A seção II introduz a técnica de sensoriamento original. As alterações feitas no método são apresentadas na subseção B. O sistema de medidas, desenvolvido para adquirir dados que validassem o método utilizado, é apresentado na seção III, assim como as configurações da medição. Na seção IV, encontram-se todos os resultados de medida, assim como as comparações entre o método original e o método proposto. Por fim, a seção V traz as conclusões do presente trabalho.

\section{TÉCNICAS DE SENSORIAMENTO}

\section{A. Método de Sensoriamento}

A técnica de sensoriamento apresentada em [4] foi a base de desenvolvimento do presente trabalho. O sensoriamento de espectro é tratado como um problema de detecção de bordas, em que há descontinuidades entre as bandas vizinhas.

O espectro, faixa de freqüências em que será feito o sensoriamento, é modelado como uma sucessão de subbandas de limites $F_{i}, i=1,2, \ldots, N$, em que $F_{i}$ representa as freqüências, em $\mathrm{Hz}$, onde ocorrem descontinuidades. A técnica consiste em identificar esses limites, ou seja, identificar as sub-bandas existentes e posteriormente estimar a energia em cada uma delas.

A densidade espectral de potência,$S_{i}(f)$, é considerada constante em cada sub-banda, $F_{i}-F_{i-1}$, ena ausência de ruído, pode ser calculada por:

$$
\int_{F_{i}-1}^{F_{i}} S_{i}(f) d f=F_{i}-F_{i-1} .
$$

A densidade espectral de potência, $S_{r}(f)$, do sinal observado $r(t)$ pode ser escrita como:

$$
S_{r}(f)=\sum_{i=1}^{N} \alpha_{i}^{2} S_{i}(f)+S_{w}(f)
$$

em que $\alpha_{i}^{2}$ é a densidade espectral de potência do sinal dentro da i-ésima banda e $S_{w}(f)$ é a densidade espectral de potência do ruído. Após a identificação das freqüências $\left\{F_{i}\right\}_{n=1}^{N-1}$, estima-se a densidade espectral de potência dentro de cada banda:

$$
B_{i}=\frac{1}{F_{i}-F_{i-1}} \int_{F_{i}-1}^{F_{i}} S_{r}(f) d f
$$

A técnica considera que dentro do espectro analisado, sempre existirá uma banda vazia, $B_{i}^{\prime}$. Estima-se, portanto, que a densidade espectral de potência dessa banda corresponde à densidade espectral de potência do ruído, $N_{0} / 2$. Dessa forma, para todas as outras bandas, estima-se $\alpha_{i}^{2}$ por:

$$
\hat{\alpha}_{i}^{2}=B_{i}-\min _{i^{\prime}} B_{i^{\prime}}, i=1, \ldots, N
$$

A identificação das descontinuidades $\left\{F_{i}\right\}_{n=1}^{N-1}$ é feita utilizando a transformada wavelet [4], A dilatação da função wavelet $\phi(f)$ por uma escala s, é dada por:

$$
\phi_{s}(f)=\frac{1}{S} * \phi\left(\frac{f}{s}\right)
$$

Para escalas diádicas, $s=2^{j}, i=1,2 \ldots J$. A transformada wavelet contínua, no domínio da freqüência, é dada por:

$$
W_{s} S_{r}(f)=S_{r} * \phi_{s}(f)
$$

O valor de $W_{s} S_{r}(f)$ para uma determinada escala $s$, representa uma medida de correlação entre a wavelet nessa escala e os valores de $S_{r}(f)$ [5]. Assumindo-se que wavelets são funções de suporte compacto, ou seja, têm um decaimento suficientemente rápido, o valor de $W_{s} S_{r}(f)$ em torno de uma freqüência $f_{0}$ depende, unicamente, dos valores de $S_{r}(f)$ em uma vizinhança de $f_{0}$, proporcional à escala s. Em pequenas escalas, a transformada wavelet provê informação localizada sobre a função $S_{r}(f)$. Dessa forma, considerando que $W_{s}^{1} S_{r}(f)$ e $W_{s}^{2} S_{r}(f)$ são, respectivamente, a derivada primeira e segunda da transformada wavelet de $S_{r}(f)$ :

$$
\begin{gathered}
W_{s}^{1} S_{r}(f)=s \frac{d}{d f}\left(S_{r} * \phi_{s}\right)(f) \\
W_{s}^{2} S_{r}(f)=s^{2} \frac{d^{2}}{d f^{2}}\left(S_{r} * \phi_{s}\right)(f)
\end{gathered}
$$

Para escalas fixas, os máximos locais de $W_{s}^{1} S_{r}(f)$ correspondem aos cruzamentos em zero de $W_{s}^{2} S_{r}(f)$ e aos pontos de inflexão de $S_{r}(f)$.

$$
\begin{gathered}
\widehat{F}_{i}=\operatorname{máximo}\left\{\left|W_{s}^{1} S_{r}(f)\right|\right\}, \quad f \epsilon\left(F_{0}, F_{N}\right) \\
\hat{F}_{i}=\operatorname{zeros}\left\{W_{s}^{2} S_{r}(f)\right\}, \quad f \epsilon\left(F_{0}, F_{N}\right)
\end{gathered}
$$

Bordas e descontinuidades propagam-se por diversas escalas s. Constrói-se, então, o produto de $J$ gradientes da transformada wavelet contínua:

$$
U_{J} S_{r}(f)=\prod_{j=1}^{J} W_{s=s_{j}}^{1} S_{r}(f)
$$

Dessa forma, o método propõe a análise multi-escala para a estimação das freqüências $\widehat{F}_{i}$, com o intuito de captar os 
picos de $W_{S} S_{r}(f)$ devido às descontinuidades de $S_{r}(f)$ e não ao ruído:

$$
\widehat{F}_{i}=\operatorname{máximo}\left\{\left|U_{J} S_{r}(f)\right|\right\}, \quad f \epsilon\left(F_{0}, F_{N}\right)
$$

A simulação apresentada em [4] utiliza a wavelet Gaussiana, com escalas $s=2^{i}, i=1, \ldots 4$.

\section{B. Método Proposto}

A transformada wavelet contínuapode ser contemplada como a correlação não normalizada entre o sinal a ser transformado e uma função wavelet-mãe $\phi(f)$ dilatada por uma escala $s$ e transladada por um fator $\tau$ [5]:

$$
W_{s} S_{r}(f)=\left\langle S_{r}(f), \phi_{s, \tau}(f)\right\rangle=C_{\phi_{s}}(s, \tau)
$$

em que $C_{\phi_{s}}(s, \tau)$ representa os coeficientes da transformada wavelet contínua. Assim, ao aplicar a transformada para uma escala $s=s_{j}$ é possível identificar os pontos de $S_{r}(f)$ mais correlacionados com $\phi_{s_{j}}(f)$ a partir dos picos de $C_{\phi_{s_{j}}}\left(s_{j}, \tau\right)$.

Como o objetivo é detectar as bordas das sub-bandas de $S_{r}(f)$, valores pequenos de $s_{j}$ devem ser escolhidos a fim de proporcionar uma menor dilatação na wavelet-mãe $\phi(f)$. Assim, para um valor pequeno de $s_{j}$, os pontos de máximo dos coeficientes $C_{\phi_{s_{j}}}\left(s_{j}, \tau\right)$ indicarão as descontinuidades em $S_{r}(f)$.

O método descrito em [4] propõe ainda derivar a expressão $C_{\phi_{s_{j}}}\left(s_{j}, \tau\right)$. Desse modo os picos de $C_{\phi_{s_{j}}}{ }^{\prime}\left(s_{j}, \tau\right)$ indicariam regiões vizinhas aos picos de $C_{\phi_{s_{j}}}\left(s_{j}, \tau\right)$ e, portanto, próximas das bordas das sub-bandas de $S_{r}(f)$. O uso da derivada tem como função mitigar as componentes ruidosas, de modo a realçar apenas picos que correspondam a tais bordas.

Contudo, essa abordagem é eficiente apenas para bordas com inclinações acentuadas. Nessas situações, como os picos de $C_{\phi_{s_{j}}}\left(s_{j}, \tau\right)$ apresentam bases relativamente pequenas, as regiões vizinhas dadas pelos pontos de máximo de $C_{\phi_{s_{j}}}{ }^{\prime}\left(s_{j}, \tau\right)$ representam uma boa aproximação das bordas de $S_{r}(f)$.

Nos casos em que as sub-bandas de $S_{r}(f)$ apresentam bordas de inclinação não muito acentuada, ou em casos com um maior nível de ruído, o método da derivada se mostra bastante ineficiente. Isso ocorre porque, em tais casos, os picos de $C_{\phi_{s_{j}}}\left(s_{j}, \tau\right)$ apresentam bases mais largas. Dessa maneira, cada máximo de $C_{\phi_{s_{j}}}\left(s_{j}, \tau\right)$ determinará picos em $\mathrm{C}_{\phi_{s_{j}}}{ }^{\prime}\left(s_{j}, \tau\right)$ que não corresponderão ao local exato da respectiva borda em $S_{r}(f)$.

Portanto, é proposto que a estimação dos limites das subbandas de $S_{r}(f)$ seja feita a partir dos picos dos coeficientes $C_{\phi_{s_{j}}}\left(s_{j}, \tau\right)$ e não dos máximos de suas derivadas. Desse modo, pode-se escrever:

$$
V_{J} S_{r}(f)=\prod_{j=1}^{J} W_{s=s_{j}} S_{r}(f)
$$

Outra alteração proposta está relacionada com as escalas utilizadas para detecção das bordas. Muitas vezes, os limites das sub-bandas de $S_{r}(f)$ não se comportam de maneira similar, podendo apresentar inclinações mais ou menos abruptas. Desse modo, ao se realizar o produto descrito em (14) para um grupo $\left\{s_{j}\right\}, j=1, \ldots, J$ de escalas é possível que bordas fracamente correlacionadas com os respectivos $\phi_{s_{j}}(f)$ gerados não sejam detectadas.

Para a solução desse problema propõe-se uma análise com múltiplos grupos de escalas $\left\{s_{j k}\right\}, j=1, \ldots, J$ e $k=1, \ldots, K$. Dessa forma, a partir de (14), pode-se estimar as bordas utilizando cada grupo $k$. Posteriormente, os resultados de todos os $K$ grupos são combinados de modo a se ter:

$$
\widehat{F}_{i}=\left\{\operatorname{máximo}\left\{\left|V_{J} S_{r}(f)\right|\right\}\right\}_{k=1}^{K} f \epsilon\left(F_{0}, F_{N}\right)
$$

\section{SISTEMA DE MEDIDAS}

Com o intuito de validar o método de deteç̧ão de energia apresentado em [4] e as alterações propostas neste artigo, criou-se um sistema para a coleta de dados. O sistema consiste em um analisador de espectro Agilent, modelo E4402B, uma antena para a faixa onde será realizado o sensoriamento e um computador notebook, que permite o controle do analisador de espectro através da interface GPIB.

O programa Spectrum_Sensing, desenvolvido na linguagem Agilent VEE Pro, permite o armazenamento dos dados obtidos em um único arquivo de texto. O controle do equipamento é feito com a utilização da Standard Commands for Programmable Instrumentation (SCPI).

A implementação funciona da seguinte forma: definem-se os parâmetros iniciais da medida do analisador de espectro, como a freqüência central, o span, o nível de referência, o tempo de cada varredura do analisador de espectro e o tempo entre as varreduras.

A freqüência inicial e o span permitem o ajuste da banda de interesse. Já o tempo de varredura e o tempo entre as varreduras, permitem ajustar o sistema de medição às características dos sistemas da banda de interesse, otimizando o processo de sensoriamento. Como a implementação é bem flexível, há a possibilidade de realizar o sensoriamento com diferentes configurações.

Após a configuração dos parâmetros, inicia-se a etapa de aquisição das medidas do analisador. Espera-se $m$ segundos, em que $m$ é o tempo entre as varreduras, e então, a varredura é iniciada. O processo dura $t$ segundos, onde $t$ é o tempo de varredura. O processo é repetido até ser interrompido pelo usuário.

Após a coleta dos dados, a técnica de sensoriamento apresentada no item II é aplicada.

\section{A. Configurações}

A técnica de sensoriamento de espectro foi testada em diversas bandas, sempre buscando adequar os parâmetros de medição, como o span e o intervalo entre as varreduras às características dos sinais de interesse. No item IV avalia-se os resultados na faixa de radiodifusão sonora em FM e em 800MHz, na faixa de uplink da banda A do serviço móvel 
celular. O plano de destinação de faixas de freqüência no Brasil pode ser consultado no site da ANATEL - Agência Nacional de Telecomunicações [12].

Para a primeira medição, ajustou-se o sistema de medidas para uma freqüência central de $98 \mathrm{MHz}$, span de $20 \mathrm{MHz}$, para que a faixa de sensoriamento fosse de $88 \mathrm{MHz}$ a 108 $\mathrm{MHz}$. Nesse caso, o tempo de varredura foi de $5 \mathrm{~ms}$ e tempo entre as varreduras de 1s, já que o objetivo era apenas identificar os limites entre os canais, e assim validar a técnica. Para a segunda medição, o sistema foi ajustado para a faixa de 824 a $835 \mathrm{MHz}$, span de $11 \mathrm{MHz}$.

\section{RESULTADOS}

\section{A. Dados simulados}

Primeiramente, foram feitas algumas simulações com o objetivo de validar o método.

A primeira simulação utiliza um modelo de espectro bem semelhante àquele proposto por [4], ilustrado na Figura 1(a). As bordas das sub-bandas apresentam uma característica abrupta e, portanto, podem ser estimadas utilizando $C_{\phi_{s_{j}}}{ }^{\prime}\left(s_{j}, \tau\right)$ através da técnica descrita em (11). Para essa simulação foram consideradas $N=6$ sub-bandas $\left\{B_{n}\right\}$ com limites nas freqüências $\left\{F_{i}\right\}_{n=0}^{6}=[150,280,365$, $385,465,510,550]$. Os níveis de densidade espectral para cada sub-banda foram fixados em $\{\hat{\alpha}\}_{n=1}^{6}=$ $[60,0,80,5,50,10]$.

A Figura 1(b) mostra os coeficientes $C_{\phi_{s_{j}}}{ }^{\prime}\left(s_{j}, \tau\right)$ para $s_{j}=[2,4,8,16]$, além do produto dado em (11). Como visto, regiões de máximo em $C_{\phi_{s_{j}}}{ }^{\prime}\left(s_{j}, \tau\right)$ indicam os pontos de descontinuidade de $S_{r}(f)$. É possível observar, ainda, como o produto definido em (11) aprimora a localização de tais pontos.

Para gerar os coeficientes $C_{\phi_{s_{j}}}{ }^{\prime}\left(s_{j}, \tau\right)$, foi escolhida a wavelet-mãe de haar. Por apresentar bordas abruptas, esse tipo de wavelet-mãe proporciona uma maior correlação do espectro $S_{r}(f)$ com as wavelets dilatadas $\phi_{s_{j}}(f)$.

Dessa maneira, obtem-se para os limites das sub-bandas e para os níveis da densidade espectral, respectivamente:

$$
\begin{gathered}
\left\{F_{i}\right\}_{n=0}^{6}=[150,280,366,385,465,510,550] \\
\{\hat{\alpha}\}_{n=1}^{6}=[60,0,78,5,46,10]
\end{gathered}
$$

Os limites $\left\{F_{i}\right\}_{n=0}^{6}$ são indicados pelas linhas pontilhada na Figura 1(a).

Para um segundo exemplo proposto, ilustrado na Figura 2(a), foi considerado um modelo de espectro bem semelhante ao anterior, mas com bordas menos abruptas. A Figura 2(b) mostra os produtos indicados em (11) e (14) respectivamente. Para essa nova situação as bordas das subbandas já não podem ser estimadas de forma correta utilizando a derivada dos coeficientes $C_{\phi_{s_{j}}}\left(s^{\prime}, \tau\right)$, como originalmente proposto em [4]. Nesse caso, os picos de $C_{\phi_{s_{j}}}\left(s^{\prime}, \tau\right)$ apresentam bases largas e, portanto, as derivadas $C_{\phi_{s_{j}}}{ }^{\prime}\left(s_{j}, \tau\right)$ já não indicam de forma precisa as descontinuidades de $S_{r}(f)$. No entanto, ao se aplicar o método proposto na seção II.B, verifica-se que tais bordas podem ser perfeitamente identificadas pelos picos do produto dos coeficientes $C_{\phi_{s_{j}}}\left(s^{\prime}, \tau\right)$, como descrito em (14).

Na Figura 2(a) é possível verificar os limites estimados utilizando o método original, proposto em [4] (linha pontilhada), e o novo método sugerido (linha contínua). Como o espectro proposto não apresenta bordas abruptas, foi escolhida uma wavelet-mãe gaussiana para gerar os coeficientes $C_{\phi_{s_{j}}}\left(s_{j}, \tau\right)$ e $C_{\phi_{s_{j}}}{ }^{\prime}\left(s_{j}, \tau\right)$, já que essa apresenta variações mais suaves.

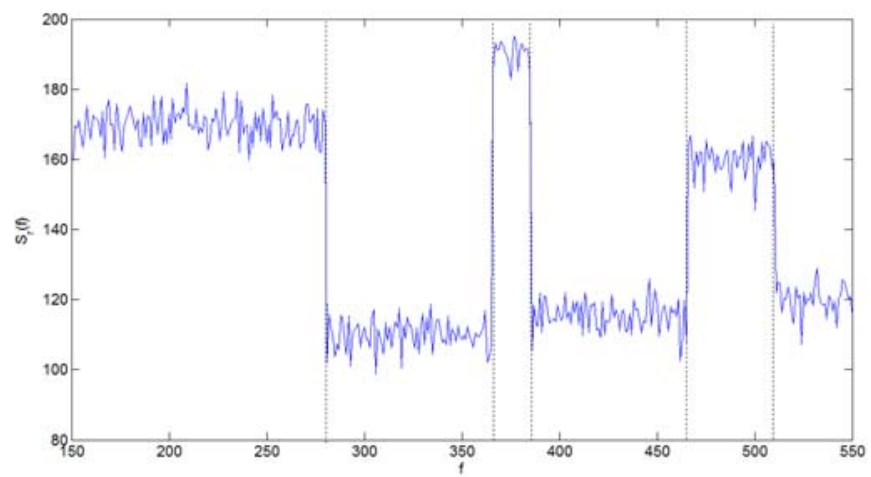

(a)

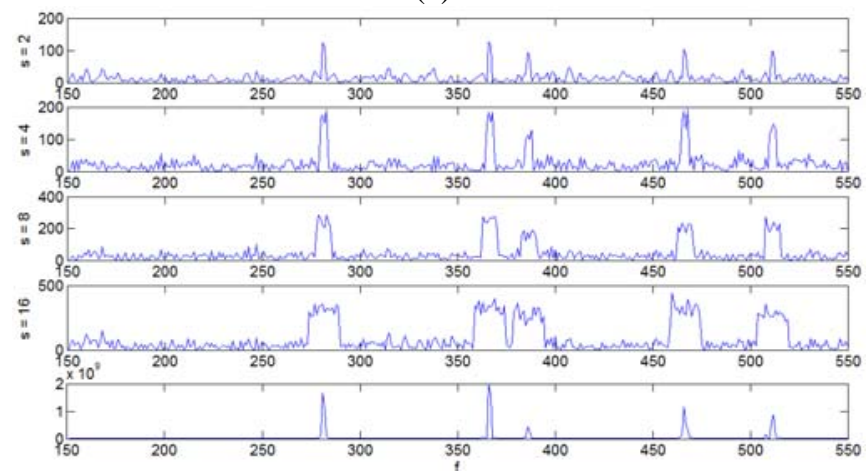

(b)

Fig. 1. (a) Densidade Espectral de Potência do sinal com localização das sub-bandas utilizando método descrito por [4]. (b) Coeficientes calculados utilizando (7) para $s_{j}=[2,4,8,16]$ e produto para múltiplas escalas (11). Para a simulação foi empregada a wavelet-mãe de haar, $\phi_{\text {haar }}$.

Finalmente, a Figura 3(a) mostra um modelo de espectro com sub-bandas limitadas por bordas com diferentes inclinações. Para essa simulação foram consideradas $N=6$ sub-bandas $\left\{B_{n}\right\}$ com limites nas freqüências $\left\{F_{i}\right\}_{n=0}^{6}=$ $[150,175,180,250,350,400,550]$. Nesse caso utilizar o método com apenas um grupo de escalas não é suficiente. Esse fato pode ser comprovado pela Figura 3(b), que mostra como cada grupo de escalas é capaz de detectar apenas algumas bordas. Já o método com múltiplos grupos oferece uma resposta bastante satisfatória, pois combina a contribuição de cada um dos grupos de escalas. Utilizando o método com múltiplos grupos, com uma wavelet-mãe do tipo gaussiana encontra-se, para os limites das sub-bandas e para os níveis da densidade espectral, respectivamente:

$$
\begin{gathered}
\left\{\widehat{F}_{l}\right\}_{n=0}^{6}=[150,174,180,251,351,436,550] \\
\{\hat{\alpha}\}_{n=1}^{6}=[0,122,6,159,29,120]
\end{gathered}
$$




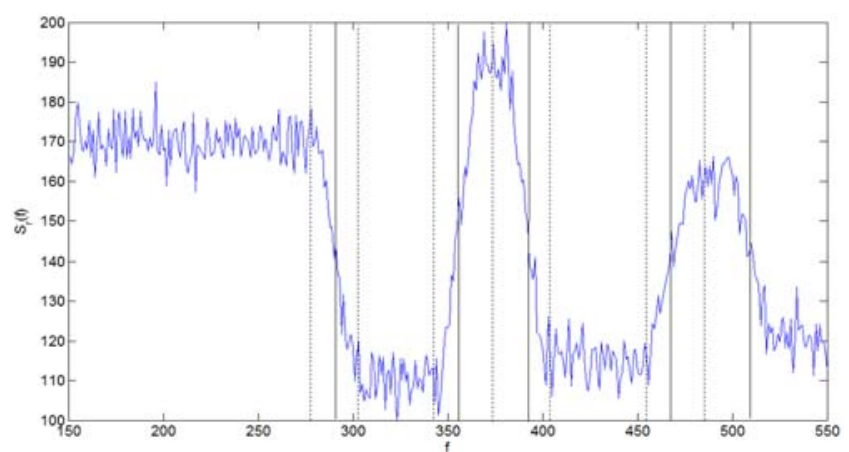

(a)
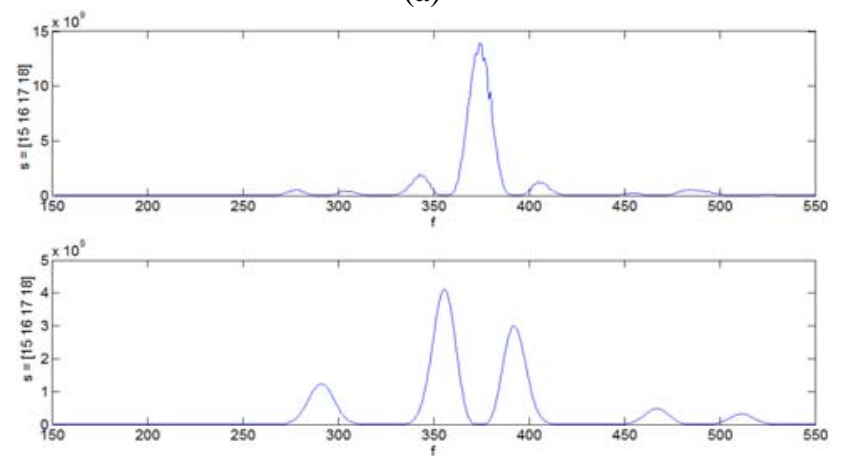

(b)

Fig. 2. (a) Densidade Espectral de Potência do sinal com localização das sub-bandas utilizando o método original proposto em [4] (linha pontilhada) e o novo método sugerido (linha contínua). (b) Produto para múltiplas escalas $s_{j}=$ $[15,16,17,18]$ utilizando (11) e (14). Para a simulação foi empregada a wavelet-mãe gaussiana, $\phi_{\text {gaus }}$.

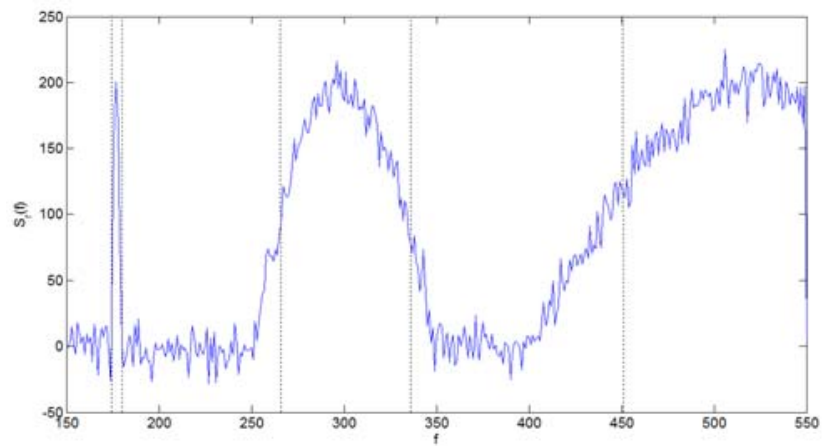

(a)

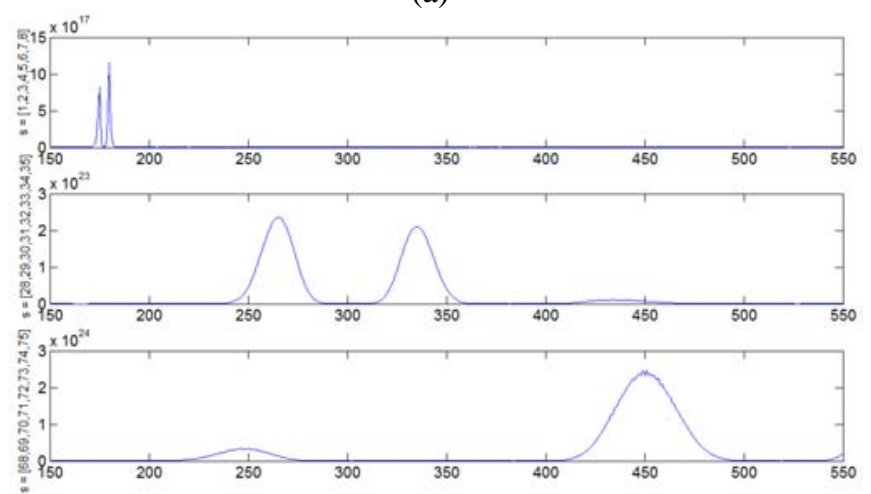

(b)

Fig. 3. (a) Densidade Espectral de Potência do sinal com localização das sub-bandas utilizando método com múltiplos grupos de escalas, descrito em (15). (b) Produtos de cada um dos grupos de escalas $s_{j k}=\{[1: 8],[28: 35],[68: 75]\}$. Para a simulação foi empregada a wavelet-mãe gaussiana, $\phi_{\text {gaus }}$.

\section{B. Dados coletados experimentalmente}

Os dados experimentais foram coletados conforme detalhado na seção III.

A primeira faixa analisada foi a de radiodifusão sonora em FM de 88 a $108 \mathrm{MHz}$. Essa faixa foi escolhida por ter um espectro conhecido, assim se pode analisar a confiabilidade da técnica.

A Figura 4(a) ilustra a divisão das sub-bandas ao se utilizar o método original descrito em [4]. Na Figura 4(b) são mostrados esses limites ao se utilizar as modificações propostas na seção II.B. Para ambos os casos foram utilizados o mesmo grupo de escalas $s_{j}=[1,2,4]$ e a mesma wavelet-mãe, do tipo gaussiana.

Na Figura 4, pode-se observar a diferença de precisão entre a técnica original [4] e a técnica proposta no artigo. Nota-se, na Figura 4 (a) que, caso a técnica original fosse utilizada para sensoriar o espectro apresentado, ela identificaria canais inexistentes, ou seja, o cálculo de energia seria feito, desnecessariamente, para bandas que não representam canais reais. Já a técnica proposta, como pode ser observado na Figura 4(b), obteve um desempenho superior, identificando com precisão os limites entre os canais ocupados no espectro. Conclui-se, portanto que apesar de ter um desempenho bom quando utilizada em situações ideais, como a da Figura 1(a), a técnica original apresenta imperfeições quando é aplicada a dados reais.

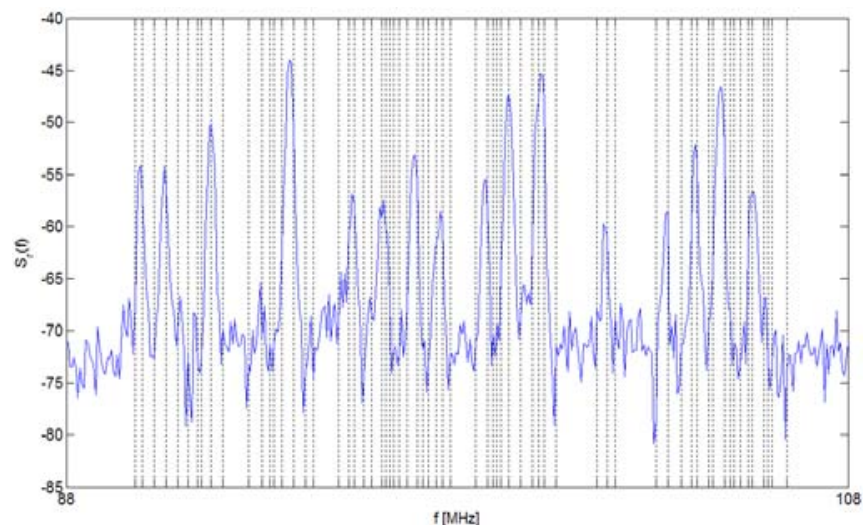

(a)

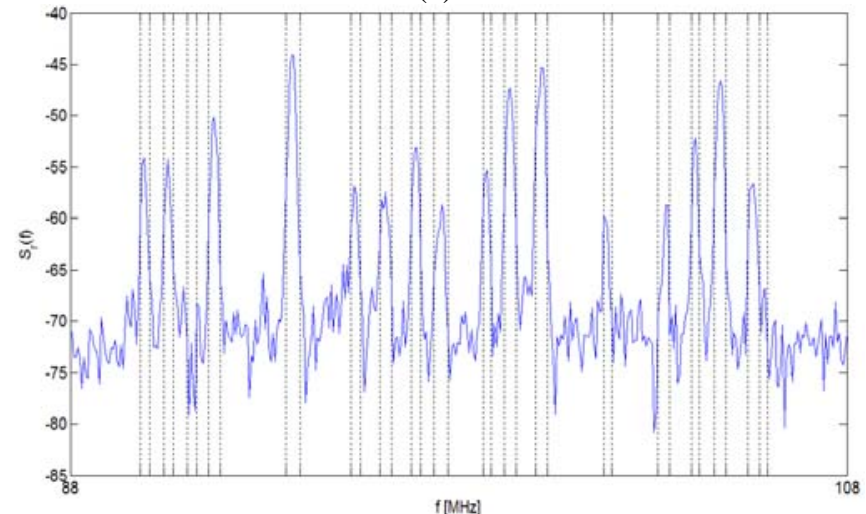

(b)

Fig. 4. (a) Densidade Espectral de Potência para faixa de radiodifusão sonora em FM (88 a $108 \mathrm{MHz}$ ). As linhas verticais ilustram a localização das sub-bandas utilizando o método original proposto em [4] (b) e o novo método sugerido por (14). Foi empregada a wavelet-mãe gaussiana, $\phi_{\text {gaus }}$. 
Em uma segunda medida foram coletados dados na faixa de 824 a 835 MHz. Tal faixa é destinada ao uplink da banda A do serviço móvel celular. A análise do método em tal banda é crucial para verificar se o mesmo pode ser utilizado para o sensoriamento de espectro em futuras redes de rádio cognitivo.

A Figura 5 mostra os limites das sub-bandas para esse espectro utilizando o método original Figura 5(a) e o proposto na seção II.B, Figura 5(b). Foram utilizadas as escalas $\quad s_{j}=[1,1.1,1.2,1.3,1.4,1.5,1.6,1.7,1.8] \quad$ e a wavelet-mãe gaussiana. Pode-se verificar que o primeiro método identifica bordas inclusive na região de ruído. Já o método proposto continua apresentando um bom desempenho para a nova faixa de freqüências.

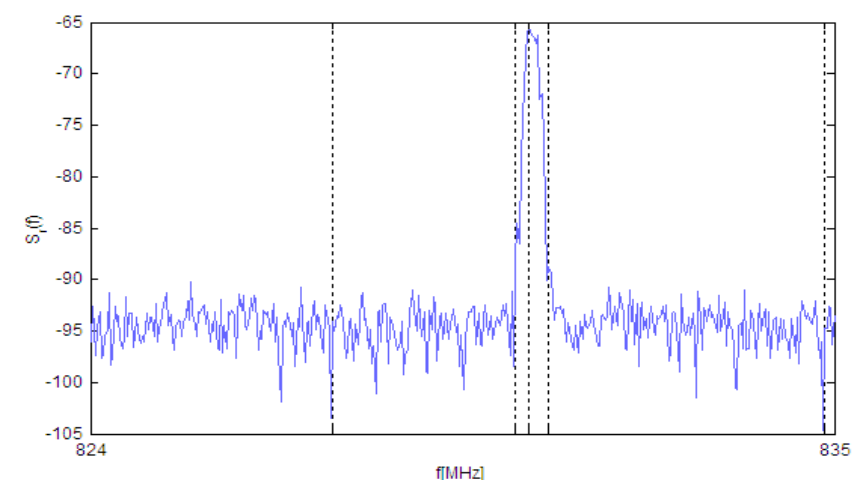

(a)

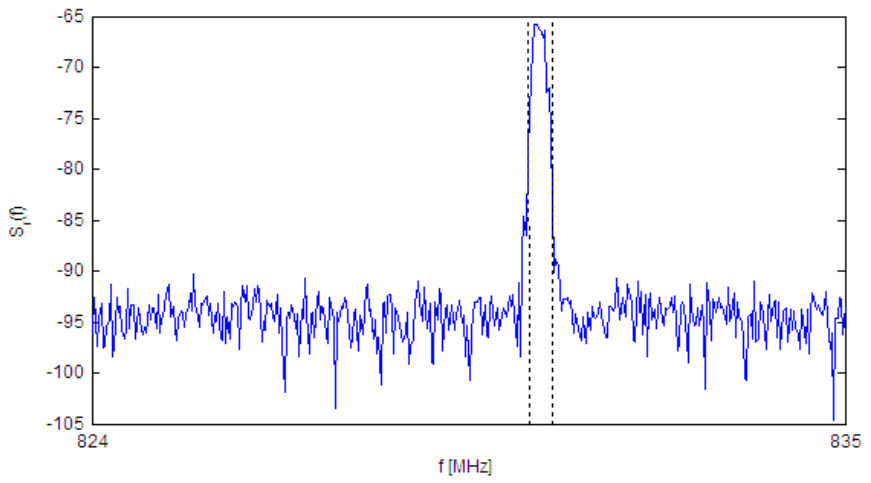

(b)

Fig. 5. (a)Densidade Espectral de Potência para faixa de uplink da banda A do serviço móvel celular (824 a $835 \mathrm{MHz}$ ). As linhas verticais ilustram a localização das sub-bandas o método original proposto em [4] (b) e o novo método sugerido por (14). Foi empregada a wavelet-mãe gaussiana, $\phi_{\text {gaus }}$.

\section{CONCLUSÃO}

$\mathrm{O}$ artigo se propôs a analisar experimentalmente uma técnica de sensoriamento de espectro baseada em detecção de energia por wavelets [4]. Para tanto, desenvolveu-se uma metodologia de medidas que permitisse validar a técnica .

Ao se avaliar o desempenho da técnica original, verificouse que, apesar de ser eficiente na detecção de descontinuidades em sinais simulados, a mesma mostrou-se pouco robusta quando utilizada em medidas reais do espectro.
O artigo apresenta alterações na técnica original, que a tornam eficaz tanto em situações simuladas quanto em situações reais. Mostram-se resultados que validam a técnica proposta em relação a medidas de espectro na faixa de radiodifusão sonora, 88 a $108 \mathrm{MHz}$ e na faixa de uplink da banda A do serviço móvel celular, 824 a 835 MHz.

\section{REFERÊNCIAS}

[1] J. Mitola III and G.Q. Maguire Jr., Cognitive Radio: Making Software Radios More Personal, IEEE Personal Communications, vol. 6, no. 4, pp. 13-18, Agosto 1999.

[2] S. Haykin, Cognitive Dynamic Systems, Proceedings of the IEEE, vol. 94, no. 11, pp. 1910-1911, Novembro 2006,

[3] I. F. Akyildiz, W.-Y. Lee, M.C. Vuran, S. Mohanty, NeXt generation/dynamic spectrum access/cognitive radio wireless networks: a survey, Computer Networks, vol. 50, no. 13, pp. 2127-2159, Setembro 2006.

[4] Z. Tian, G. B. Giannakis, A Wavelet Approach to Wideband Spectrum Sensing for Cognitive Radios, $1^{\text {st }}$ International Conference on Cognitive Radio Oriented Wireless Networks and Communications, 2006., pp. 1-5, Junho 2006.

[5] S. Mallat e W. L. Hwang, Singularity Detection and processing with Wavelets, Information Theory, IEEE Transactions on, volume 38, pp. 617 - 643, Março 1992.

[6] H. Urkowitz, Energy detection of unknown deterministic signals, Proceedings of the IEEE, vol. 55, no. 4, pp. 523-531, Abril 1967.

[7] A. V. Dandawate and G. B. Giannakis, Statistical tests for presence of cyclostationarity, IEEE Transactions on Signal Processing, vol. 42, no. 9 pp. 2355-2369, Setembro 1994.

[8] J. Lunden, V. Koivunen, A. Huttunen, and H. V. Poor, Spectrum Sensing in Cognitive Radios Based on Multiple Cyclic Frequencies, CROWNCOM, 2007.

[9] D. Cabric, A. Tkachenko e R. W. Brodersen, Experimental Study of Spectrum Sensing based on Energy Detection and Network Cooperation, ACM International Conference Proceedings, Vol. 222, no. 12, 2006.

[10] S. Haykin, Cognitive Radio: Brain Empowered Wireless Communciations, IEEE J. Select. Areas Commun, vol. 23, no. 2, pp. 201220, Fevereiro 2005.

[11] Daubechies, Ten Lectures on Wavelets. Phliadelphia, PA: SIAM, 1992.

[12] Plano de destinação de Faixas de freqüência. Disponível em: http://sistemas.anatel.gov.br/pdff/. Acesso em 15 jun 2008. 